\title{
Process Algebra and Non-interference
}

\author{
P Y A Ryan \\ Defence Evaluation and Research Agency \\ St Andrews Road, Malvern, UK
}

\author{
S A Schneider \\ Royal Holloway, University of London \\ Egham, Surrey, UK, TW20 0EX
}

\begin{abstract}
The information security community has long debated the exact definition of the term 'security'. Even if we focus on the more modest notion of confidentiality the precise definition remains controversial. In their seminal paper [4], Goguen and Meseguer took an important step towards a formalisation of the notion of absence of information flow with the concept of non-interference. This too was found to have problems and limitations, particularly when applied to systems displaying non-determinism which led to a proliferation of refinements of this notion and there is still no consensus as to which of these is 'correct'.

We show that this central concept in information security is closely related to a central concept of computer science: that of the equivalence of systems. The notion of noninterference depends ultimately on our notion of process equivalence. However what constitutes the equivalence of two processes is itself a deep and controversial question in computer science with a number of distinct definitions proposed in the literature. We illustrate how several of the leading candidates for a definition of non-interference mirror notions of system equivalence. Casting these security concepts in a process algebraic framework clarifies the relationship between them and allows many results to be carried over regarding, for example, composition and unwinding.

We also outline some generalisations of non-interference to handle partial and conditional information flows.
\end{abstract}

\section{Introduction}

It is a source of mild embarrassment that the information security community has not yet reached a consensus as to the precise meaning on the term 'security' or even to the simpler question of what is meant by confidentiality. It is clear that confidentiality boils down to the absence of certain undesirable information flows, but this begs the question of what constitutes an information flow or its absence.

(C)IEEE. Appeared in Proceedings of CSFW 12.
The thesis of this paper is that the characterisation of the absence of information flow reduces to characterising the equivalence of systems. The latter is of course a central concept in computer science and here too we find that there is no single agreed definition of what constitutes equivalence. Instead we again find a proliferation of definitions and it seems that which is appropriate depends on the particular application. Indeed, as we will show in detail in the later sections of this paper, there is a close correspondence between formulations of non-interference and formulations of process equivalence. It appears that by and large the proponents of the various forms of non-interference were unaware of the analogies with notions in the process algebra community and were independently reinventing these concepts in the security context.

A number of attempts to unify the various formulations of non-interference have been published recently, notably McLean [8] and Foccardi and Gorrierri [2]. These are very elegant and illuminating. We believe that identifying the analogies between the concepts in the security and the process algebra communities sheds further light on the notion of confidentiality and in particular helps explain the proliferation of definitions of confidentiality. Indeed the security community can perhaps derive some solace from the observation that the problem of defining non-interference is equivalent in difficulty to one of the central problems of computer science and so it should not be a source of surprise that consensus has eluded us.

Process algebras, in particular CSP, provide a general framework for describing interacting systems. Many of the formulations of confidentiality that one finds in the literature are presented in rather specialised and often specially tailored models of computation, so recasting them in a process algebra allows them to be generalised and compared. Another advantage of using a process algebraic framework is that it allows us to apply a number of established results, such as the completeness of unwinding rules, and compositionality.

We start with a brief overview of those aspects of CSP that we use in this paper. We then present a number of formulations of non-interference in a CSP style. In Sec- 
tion 4 we discuss some generalisations of non-interference designed to encompass a richer class of security policies.

\section{Process algebra}

Process algebras provide a particular approach to the study of concurrency and interaction. This paper bases its discussion within the framework of the process algebra CSP (Communicating Sequential Processes). A full account of this process algebra can be found in $[11,15]$. It provides a language for describing interacting systems, together with a semantic theory for understanding them. This section provides a brief reminder of those aspects most relevant to this paper.

The language of CSP is constructed around events: instantaneous synchronisations which provide the communication primitive. Events may have some structure, the most common communication being a channel communication of the form $c . v$, where $c$ is the channel name, and $v$ is the value communicated. For the purposes of this paper we will divide $\Sigma$, the set of all events, into two classes: $H$ (high) and $L$ (low). The set $H$ will be further divided into high inputs $H I$ and high outputs $H O$.

Processes are used to describe possible patterns of interaction. In CSP, a process has an alphabet, or interface: the set of events it is able to synchronise on. The process $c ! v \rightarrow P$ describes a process which is prepared to output $v$ on channel $c$, and behave subsequently as $P$. The input $c ? x \rightarrow P(x)$ may take in some value $v$ along channel $c$ and behave subsequently as $P(v)$. The choice $P \sqcap Q$ may behave non-deterministically either as $P$ or as $Q$. Processes may be put in parallel: $P \| Q$ behaves as $P$ running concurrently with $Q$, synchronising on events in their common alphabets, and performing other events independently. An interleaving of two processes, $P \| \mid Q$, simply executes $P$ and $Q$ concurrently without any communication occurring between them. Values are passed between parallel processes by means of synchronisations on the channels, linking an output channel of one to an input channel of another. The abstraction mechanism $P \backslash A$ describes the process $P$ with all occurrences of $A$ occurring internally in the resulting process. The process $S T O P_{A}$ has alphabet $A$ and can perform no events at all. Thus $P \| S T O P_{A}$ behaves as the process $P$ with all occurrences of $A$ blocked. This is different from $P \backslash A$ in which all occurrences of events in $A$ are made internal.

The process $R U N_{A}$ also has alphabet $A$, and it is always ready to perform any event from the set $A$. The process $C H A O S_{A}$ also has alphabet $A$, and can perform or refuse to perform any such event at any stage during an execution-it is the most nondeterministic (divergence-free) process with alphabet $A$. Processes may also be recursively defined, by giving equations which contain the name of the process be- ing defined as a subterm of the process expression. For example, the process

COPY $=$ in $? x \rightarrow$ out $t x \rightarrow C O P Y$

defines a buffer process $C O P Y$ as one which repeatedly alternates input and output. Indexed processes may also be recursively defined using families of equations.

The semantics of processes are given in terms of observations. A process is identified with the set of behaviours that may possibly be observed of it, where the kind of behaviour considered determines the nature of the model.

The Traces Model is concerned with the traces of a process: the (finite) sequences of events that it can perform during some execution. For example,

$$
\begin{aligned}
& \operatorname{traces}(\mathrm{COPY})= \\
& \quad\{\langle\rangle,\} \\
& \quad \cup\{\langle\text { in.v }\rangle \mid v \in V\} \\
& \cup\{\langle\text { in.v, out.v }\rangle \mid v \in V\} \\
& \cup\{\langle\text { in.v, out.v, in.w }| v \in V, w \in V\} \\
& \quad \vdots
\end{aligned}
$$

where $V$ is the type of the channels in, out.

If $t r$ is a possible trace of a process $P$, then $P / t r$ (pronounced ' $P$ after $t r$ ) denotes the process that $P$ becomes after executing the trace $t r$. For example, $C O P Y /\langle i n .7\rangle=$ out $7 \rightarrow$ COPY.

The traces model is sufficient for many applications but it is not able to fully distinguish different non-deterministic behaviours. To deal with non-determinism we must turn to the failures model of CSP: a failure of a process is a trace together with a refusal set which describes a set of events that the process might refuse to engage in after performing the trace. For example, $(\langle i n .3\rangle,\{i n .5, i n .6\})$ is a failure of $C O P Y$. A divergence of a process is a trace after which it may perform an infinite sequence of internal actions. In this paper, we are assuming that the systems modelled do not diverge.

A process is deterministic whenever, given an arbitrary trace $t r^{\frown}\langle a\rangle$, it could not have refused the last event instead of performing it: $(t r,\{a\})$ should not be a failure. Thus given any trace, there is only one possible response for each potential next event. In the failures model a process $Q$ is a refinement of another process $P$ when the set of $Q$ 's failures are a subset of $P$ 's failures. This means that $Q$ is more deterministic. Thus $\mathrm{CHAOS}_{A}$ is refined by any process with alphabet $A$, and deterministic processes cannot be further refined: no process is a strict refinement of a deterministic process.

Processes are considered equivalent in a semantic model if they have the same set of behaviours in that model. Thus 
if $P$ and $Q$ have the same traces, then they are equivalent in the traces model, written $P=$ traces $Q$. This means that if only their traces are examined, then they cannot be distinguished. Similarly, if $P$ and $Q$ have the same failures, then this is written $P=$ failures $Q$. The subscript to the equality symbol can be dropped if it is clear from the context.

Process semantics may also be described in terms of operational semantics, which describe transitions between states (process descriptions). Thus $P \stackrel{\mu}{\longrightarrow} P^{\prime}$ describes the performance by $P$ of a single $\mu$ event, reaching the state $P^{\prime}$. If $s$ is a sequence of events, then $P \stackrel{s}{\Longrightarrow} P^{\prime \prime}$ describes a sequence of steps between successive processes, each step labelled by the corresponding event in the sequence $s$. Operational semantics allows alternative approaches to comparing processes.

One approach to comparing processes is in terms of testing. A test $T$ is a particular kind of process, with some SUCCESS states. We consider the execution of $T$ in conjunction with a process $P$ and if $P \| T$ can reach a success state then ' $P$ may $T$ '. If $P$ may $T$ whenever $Q$ may $T$ and vice versa for all possible tests $T$, then $P$ and $Q$ are equivalent under may testing. For further information on testing see [5].

Strong bisimulation [9] identifies processes if the states in their execution graphs match. This will be the case if there is a symmetric bisimulation relation $R$ such that if $P R Q$ and $P \stackrel{\mu}{\longrightarrow} P^{\prime}$ then $\exists Q^{\prime} .\left(P^{\prime} R Q^{\prime} \wedge Q \stackrel{\mu}{\longrightarrow} Q^{\prime}\right)$, where $\mu$ is any transition label. This is the strongest useful equivalence between processes, and implies equivalence in any of the CSP semantic models. As well as requiring traces to match, bisimulation also requires the points at which choices are made within processes to match.

This can be relaxed by not requiring the two processes to match hidden $(\tau)$ events. Here we allow a visible event $a$ in $P$ to be matched by a sequence $\hat{a}$, for some $\hat{a} \in \tau^{*} . \alpha \cdot \tau^{*}$, the set of all sequences of $\tau$ actions interleaved with a single $a$. This is known as weak-bisimulation.

$P$ and $Q$ are weakly bisimilar with respect to the symmetric relation $\approx$ if and only if whenever $P \approx Q$ then $P \stackrel{a}{\longrightarrow} P^{\prime} \Rightarrow \exists Q^{\prime} \cdot Q \stackrel{\hat{a}}{\Longrightarrow} Q^{\prime} \wedge P^{\prime} \approx Q^{\prime}$.

In [3] Gardiner proposes a further weakening introduces an equivalence relation on the power set of states, known as power-bisimulation. This allows sets of states of $P$ to be related to sets of states of $Q$. This has the effect of abstracting out the effect of the point at which non-deterministic choices are made. This allows the construction of a form of bisimulation that is precisely equivalent to failures equivalence and will be discussed more fully in section 3.3.

\section{Non-interference}

\subsection{Goguen/Meseguer}

Confidentiality policies are concerned with restricting classes of information flows. In the early days of the subject such policies were typically constructed by analogy with the paper world and so involved assertions such as: information should not flow from an object of higher classification to one of lower classification. Thus information flows are treated in a binary fashion: it is either allowed to flow or not, and the objects of the policy were typically fairly gross: data bases, files, agents etc. Such analogies now look rather dated in the light of the capabilities provided by current information processing systems and we will address the question of constructing more elaborate policies, with finer granularity of objects and more subtle controls of information flows, in Section 4. For the moment we will stick to this traditional viewpoint as, even in this comparatively simple context, there are a number of subtleties to be considered.

A central problem is to characterise the absence of information flow between objects, which in effect means across interfaces or along channels. The Bell and La Padula model takes the notions of read and write as primitives and so makes no attempt to formalise them. A number of critiques of Bell and La Padula are based on this observation. An early attempt to formalise the absence of information flow was the concept of non-interference proposed in the seminal paper by Goguen and Meseguer, [4].

Intuitively the idea is as follows: to establish that information does not flow from object $A$ to object $B$ it is sufficient to establish that A's behaviour has no effect on what B can observe. Put differently: B's view of the system is independent of A's behaviour. This latter suggests an appropriate way of capturing this mathematically: for any pair of behaviours of the system that differ only in A's behaviour, B's observations of the system cannot distinguish these two systems. This makes it clear that the notion of indistinguishability of behaviours is central. For systems that are deterministic it is fairly straightforward to make such equivalence precise and indeed it is deterministic systems that Goguen and Meseguer originally considered.

Casting the Goguen/Meseguer formulation into a more CSP like notation for ease of comparison with later formulations:

Assume that High interacts with the system $S$ via the interface $\mathrm{H}$ and Low via the interface $\mathrm{L}$ and further that these two interfaces partition the full interface of $S$. Then High is said to be non-interfering with Low via $S$ if:

$$
\begin{aligned}
& \forall t r: I^{*}, c: I . \\
& \quad \text { Output }_{L} S(t r, c)=\text { Output }_{L} S(t r\lceil L, c)
\end{aligned}
$$

where $I$ is the set of inputs to $S$, and $I^{*}$ the set of sequences 
of I's. Output $t_{L} S(t r, c)$ denotes the output to Low from the system when it is in the state resulting from the sequence of inputs $t r$ and when it receives a further input $c$. Finally, the projection to $L \operatorname{tr}\lceil L$ is the trace $t r$ with all occurrences of $H$ events removed (given that $H$ and $L$ partition the alphabet of $S$ ).

In other words, whatever inputs High has performed the output that Low sees is the same as he would see if High had done nothing. Note that for a system in which the Low output is uniquely determined this equality is straightforward to define.

Even in this seemingly straightforward definition a number of subtleties lurk, for example: it depends on drawing a distinction between 'inputs' and 'outputs' without making the semantics of this distinction clear. This is a bit like the failure of the Bell La Padula model to give a precise semantics to the terms 'read' and 'write'.

More significant is the point that for systems displaying non-determinism characterising the equality of Equation 1 turns out to be rather delicate. Thus the seemingly innocent phrase '...B's observations of the system cannot distinguish...' actually conceals some subtle problems. The question of deciding when two processes should be regarded as equivalent is a difficult one and one to which a number of answers have been proposed. It is far from clear which of these is to be regarded as 'correct'. Indeed it seems reasonable to suppose that there is in fact no 'correct' notion; which is appropriate depends on the context and application in question.

The diversity of notions of system equivalence shows up most clearly in the process algebra community where we find, for example, traces or failures equivalence, various flavours of bi-simulation as well as various forms of testing equivalence.

\subsection{CSP Formulations}

In an attempt to resolve the problems associated with drawing input/output distinctions as well as address the issue of non-deterministic systems, one of the authors proposed a recasting of the Goguen/Meseguer formulation into CSP [14]. Again the notation is tweaked slightly from the original 1990 presentation to make it more compatible with the rest of this paper.

$$
\begin{aligned}
& \forall t r, t r^{\prime}: \operatorname{traces}(S) \cdot\left(t r \approx t r^{\prime} \Rightarrow\right. \\
& \left.\quad \operatorname{refusals}(S / t r) \cap L=\operatorname{refusals}\left(S /\left(t r^{\prime}\right)\right) \cap L\right)
\end{aligned}
$$

where $t r \approx t r^{\prime} \Leftrightarrow t r \mid L=t r^{\prime}\lceil L$. refusals $(S / t r)$ denotes the sets of events the process $S$ can refuse after the trace tr. A refusal is a set of events that the process may choose to refuse if the environment offers one of them. Different refusals sets can result after a given trace corresponding to different non-deterministic choices, hence the set of sets.
It thus encodes the non-determinism of a system. $\operatorname{tr}\lceil L$ denotes the restriction of the trace $t r$ to the set $L$. We take the distributed intersection of the refusal sets with $L$ to get the Low level view.

CSP does not draw a distinction between inputs and outputs, both are regarded simply as 'events'. There is a danger in wrongly categorising events as input or output which is avoided, though arguably at the cost of a characterisation of confidentiality that errs towards being too strong. A simple example, due to Millen, serves to illustrate this.

Consider a system with high and low inputs but only high outputs. Naively we would regard such a system as secure and yet it could fail the CSP characterisation. Whether or not this system really is secure depends critically on the semantics of the term 'input'. If 'input' is taken to mean an event wholly under the control of the environment that cannot be refused or delayed by the system then we probably would accept this system as being secure. However if there is any possibility of the system influencing the occurrence (even if not the value) of the 'input' event in any way we immediately have a channel from high to low. Any event that is wrongly characterised as an input in this sense could slip through and lead to a system incorrectly being deemed secure.

The CSP approach is thus a much safer criterion but could lead to some secure systems being rejected. If you are to draw such input/output distinctions and use them in the definition of security then they must be precisely defined.

In fact it turns out that, although CSP doesn't draw such distinctions, we can nonetheless use the framework, given in equation 2, to distinguish at least the High (abstracted) input/output events.

This ability to distingiush inputs/outputs in CSP arises from our use of the more symmetric formulation of [14] instead of the more traditional form in which an arbitrary trace is compared to its purge. A consequence of this is that in this formulation it is not guaranteed that the purge of an arbitrary trace is itself a valid trace of the system. At first glance this appears to be a flaw as it seems to allow Low to deduce in some cases the occurrence of certain events at the high level. It actually turns out to be an advantage: the failure of the purge of a trace to be a trace is due to the occurrence of high signal events, i.e. events that cannot be refused or delayed by High. An example of such an event might be an alarm signal to alert High of some low-level activity. This clearly does not constitute a flow from High to Low and yet Low can deduce that such an alarm event has occurred.

For example, the process

$S=l \rightarrow h \rightarrow l_{2} \rightarrow S T O P$

has traces $\left\{\langle\rangle,\langle l\rangle,\langle l, h\rangle,\left\langle l, h, l_{2}\right\rangle\right\}$. The purge $_{H}$ of $\left\langle l, h, l_{2}\right\rangle$ is $\left\langle l, l_{2}\right\rangle$ which is not a trace of $S$. Thus when Low sees $l_{2}$ 
he knows $h$ has occurred, but if High has no control over the occurrence of $h$ it does not provide a means for High to signal to Low.

If we do not want $h$ to be modelled as a signal event we should use the process

$$
\begin{aligned}
S= & l \rightarrow h \rightarrow l_{2} \rightarrow \text { STOP } \\
& \square \\
& l \rightarrow l_{2} \rightarrow \text { STOP }
\end{aligned}
$$

which allows High to refuse $h$ without deadlocking the system. Now when Low sees $l_{2}$ he cannot tell if $h$ has occurred.

Using the purge formulation would be equivalent to assuming that all events are refusable.

The issue of 'input totality' that was a concern for many of the early formulations is another manifestation of this difficulty, i.e. the problem of what happens if an invalid input sequence is presented to the system. This is dealt with automatically in a CSP approach: invalid inputs are simply refused by the system.

The approach of [14] deliberately tried to stay as close as possible to the spirit of the original Goguen/Meseguer formulation. In particular it sticks to quantifying over traces and comparison of the next events. It is interesting to consider how one might alter it to cast it in a more conventional CSP style. Firstly rather than just comparing next events we can compare the subsequent behaviours, i.e. assert equivalence as processes and use the conventional CSP hiding operator rather than having to use (distributed) set intersection:

$$
\begin{aligned}
& \forall t r, t r^{\prime}: \operatorname{traces}(S) \cdot t r \approx t r^{\prime} \Rightarrow \\
& \quad\left(( S / t r ) \left\lceilL=\text { failures }\left(S / t r^{\prime}\right)\lceil L)\right.\right.
\end{aligned}
$$

where $S\lceil L$ is the projection of the set of failures of $S$ to the set $L$ : each failure $(t r, A)$ is projected to $(\operatorname{tr}\lceil L, A \cap L)$.

In fact we can think of the earlier formulation as a kind of partial unwinding of this. Thus we can perform an inductive proof that Equation 2 implies Equation 3. It is also immediate that Equation 3 implies Equation 2, and hence that the two equations give the same characterisation.

In order to avoid the need to explicitly quantify over traces we might try expressing a characterisation directly as a CSP equivalence:

$$
S \backslash H=_{\text {failures }}\left(S \| S T O P_{H}\right) \backslash H
$$

At first glance one might think that this is equivalent to Equation 3. Putting STOP in parallel with $S$ over the alphabet $H$ has the effect of preventing all traces with $H$ events. We thus appear to be asserting that the process with all the $H$ events enabled is equivalent to that with $H$ events prevented. In fact it differs in some subtle but significant senses. Firstly this is really the purge formulation and so, in particular, it implies that the purge of any trace is itself a trace. Furthermore, the standard CSP semantics of such an equation asserts equality over stable states, that is states that cannot perform any internal (i.e. high level) events. For any state in which internal events can occur the refusal set might alter as the result the occurrence of such these transitions. It is therefore rather hard to make sense of the equality of refusal sets over unstable states, hence the decision to deal just with stable states in the standard CSP semantics. The quantification of Equation 3 however does force equality for all traces, not just traces leading to stable states (i.e. traces that can not be immediately extended by tau events). Equation 3 is therefore strictly stronger that Equation 4.

For example, the process

$S=h \rightarrow S T O P \square(l \rightarrow S T O P \sqcap S T O P)$

meets Equation 4. On the other hand it fails Equation 3, since its failures after \langle\rangle are not the same as its failures after $\langle h\rangle$, despite the low level views of both these traces being the same.

It turns out that we can cast Equation 3 in a form resembling Equation 4 and equivalent to it by using a different approach to concealing the High events. In Equation 3 we have concealed them by the rather obvious device of simply hiding them. An alternative is not to hide them but to camouflage them:

$$
S|| R U N_{H} \quad=_{\text {failures }}\left(S \| S T O P_{H}\right) \| \mid R U N_{H}
$$

Here we interleave the two systems with $R U N_{H}$ on the high level events. Now whenever Low sees an $H$ event he cannot tell if it was performed by $S$ or by $R U N_{H}$. By avoiding the use of hiding we have side-stepped the restriction to stable states. This form of abstraction was introduced by Roscoe et al [12], in which Equation 5 is shown to be equivalent to Equation 3.

The use of hiding to abstract certain events in this way provides us with another way of modelling signal events. Roscoe et al refers to this form of abstraction as 'eager' to reflect the idea that events abstracted in this way are thought of as occurring at the earliest opportunity. Correspondingly the interleaving abstraction is referred to as 'lazy'. These forms of abstraction have also been characterised in a testing framework [16] which has the added advantage of allowing input/output distinctions to be drawn over the lowlevel events as well as the high-level.

These abstractions and variants of them are discussed in detail in chapter 12 of [11].

\subsection{Unwinding}

It is usual when presenting a notion of non-interference to also present a so-called unwinding result. The idea is 
to present some constraints on the transitions of the system that together are equivalent to, or at least imply, the original property. Proving that a system obeys such constraints is more tractable than showing that it satisfies the original property.

In accordance with this tradition [14] presents such unwinding rules.

- Rule 1:

$$
\begin{aligned}
& \forall Y_{i}, Y_{j}: \operatorname{States}(S) . Y_{i} \approx Y_{j} \\
& \quad \Rightarrow \quad \operatorname{Refusals}\left(Y_{i}\right) \backslash H=\operatorname{Refusals}\left(Y_{j}\right) \backslash H
\end{aligned}
$$

- Rule 2:

$$
\begin{aligned}
& \forall Y_{i}, Y_{j}: \operatorname{States}(S) \cdot Y_{i} \approx Y_{j} \\
& \wedge \forall e: \text { Initials }\left(Y_{i}\right), e *: \text { Initials }\left(Y_{j}\right) . \\
& \quad e\left\lceil L=e *\left\lceil L \Rightarrow Y_{i} /\langle e\rangle \approx Y_{j} /\langle e *\rangle\right.\right.
\end{aligned}
$$

We have introduced an equivalence relation over states of the system. The first rule asserts that for two equivalent states Lows view of the possibilities for the next step of the system is identical.

The second rule has the effect of ensuring that the equivalence is exactly that induced by purging High events. That is, if we let $Y_{i}$ denote a state reached after trace $t r_{i}$, then rule 2 implies: $t r_{i}\left\lceil L=t r_{j}\left\lceil L \Rightarrow Y_{i} \approx Y_{j}\right.\right.$.

Proving that these imply the original non-interference is a fairly straightforward induction style proof. Showing that these are also necessary is a bit more delicate and this is proved in a rather cumbersome fashion in an appendix of [14].

A far more elegant and instructive proof of completeness can be obtained by noticing that these rules bear a remarkable resemblance to a statement of bi-simulation equivalence as used in process algebras.

In fact bi-simulation is subtly stronger than failures equivalence as it insists on the bi-similarity of individual states and draws distinctions between processes on the basis of where non-determinism is resolved. A simple example (pictured in Figure 1) illustrates this:

$$
\begin{aligned}
& P=(a \rightarrow b \rightarrow \text { STOP }) \sqcap(a \rightarrow c \rightarrow S T O P) \\
& Q=a \rightarrow(b \rightarrow S T O P \sqcap c \rightarrow S T O P)
\end{aligned}
$$

The non-deterministic choice is made earlier in $\mathrm{P}$ than in $\mathrm{Q}$ and as a result it is easy to show that no bisimulation relation can be found for them. However an environment that can only observe the $a, b$ and $c$ events cannot distinguish between them. They are failures equivalent and testing equivalent.

Gardiner, [3], introduces the notion of a power bisimulation specifically to address this point and constructs a
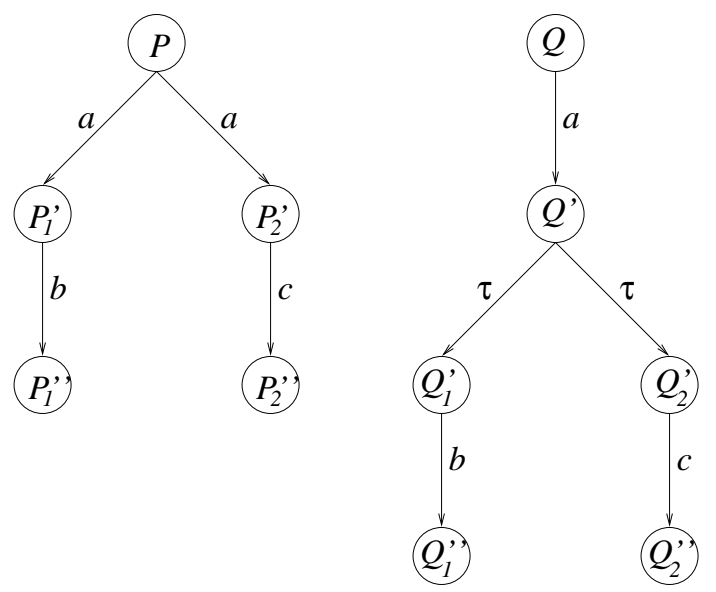

Figure 1. Processes $P$ and $Q$

bisimulation-like equivalence that is exactly as discriminating as failures equivalence. [3] gives an extremely elegant formulation and proof in terms of predicate transformers.

We notice that the unwinding rules already use the notion of an equivalence relation on the power set of states suggesting that we may be able to find a power bisimulation relation equivalent to them.

We proceed as follows: firstly we introduce what we call a 'loose-bisimulation'. This is in fact entirely analogous to weak-bisimulation except that here we require matching on $\tau$ events and treat the High events as abstracted (hidden) events. Thus we require the two processes to stay in step on the Low and $\tau$ events but allow arbitrary interleaving with high events.

We define the relation $\sim_{S}$ on states of $S$ induced by purging the $H$ events (but leaving $L$ and $\tau$ 's).

$$
\begin{aligned}
\sim_{S}=\left\{\left(S / t r, S / t r^{\prime}\right) \mid\right. & t r, t r^{\prime}: \operatorname{traces}(S) \wedge \\
& \operatorname{tr}\left\lceil L_{\tau}=t r^{\prime} \mid L_{\tau}\right\}
\end{aligned}
$$

where $L_{\tau}=L \cup\{\tau\}$

Given a state $S$ and an event $a$ define $[S]_{a}$ as:

$$
[S]_{a}=\left\{S^{\prime}: \operatorname{States}(S) \mid \exists \hat{a} \in H^{*} \cdot a \cdot H^{*} \cdot S \stackrel{\hat{a}}{\Rightarrow} S^{\prime}\right\}
$$

where $H^{*} . a H^{*}$ is the set of sequences of elements of $H$ interleaved with $a$.

We now assert that $S$ is loosely-bisimilar with respect to $\sim_{S}$ if and only if for all $a$

$$
S_{1} \sim S_{2} \Rightarrow\left[S_{1}\right]_{a} \sim\left[S_{2}\right]_{a}
$$

We now define a power bisimulation relation $\approx_{S}$ induced by purging $H$ 's and $\tau$ 's:

$$
\approx_{S}=\left\{\left(\left\{S^{\prime} \mid S \stackrel{t r}{\Longrightarrow} S^{\prime}\right\},\left\{S^{\prime} \mid S \stackrel{t r^{\prime}}{\Longrightarrow} S^{\prime}\right\}\right)\right.
$$




$$
\begin{aligned}
& \mid t r, t r^{\prime}: \operatorname{traces}(S) \wedge \\
& \operatorname{tr}\left\lceil L=t r^{\prime}\lceil L\}\right.
\end{aligned}
$$

and for $S$ and $a$ define $\langle[S]\rangle_{a}$ by:

$$
\langle[S]\rangle_{a}=\left\{S^{\prime}: \operatorname{States}(S) \mid \exists \tilde{a} \in H_{\tau}^{*} \cdot a \cdot H_{\tau}^{*} \cdot S \stackrel{\tilde{a}}{\Longrightarrow} S^{\prime}\right\}
$$

We can then easily prove the following:

Lemma 3.1 If $S$ satisfies the loose-bisimulation then it satisfies a power-bisimulation with respect to $\approx_{S}$ :

$S_{1} \approx_{S} S_{2} \Rightarrow\left\langle\left[S_{1}\right]\right\rangle_{a} \approx\left\langle\left[S_{2}\right]\right\rangle_{a}$

We can further prove the following:

Lemma 3.2 If $S_{1} \approx_{S} S_{2}$ then for all $s_{1}: S_{1}$ there exists $s_{2}: S_{2}$ such that $s_{1} \sim_{S} s_{2}$, and vice versa.

\section{Lemma 3.3}

$s_{1} \sim_{S} s_{2} \Rightarrow \operatorname{AcceptSet}_{L}\left(s_{1}\right)=\operatorname{AcceptSet}_{L}\left(s_{2}\right)$

These two lemmas prove the following:

\section{Lemma 3.4}

$S_{1} \approx S_{2} \Rightarrow \operatorname{AcceptSet}_{L}\left(S_{1}\right)=\operatorname{AcceptSet}_{L}\left(S_{2}\right)$

It is worth examining this result more carefully. We see that there are two sources of potential non-determinism in Low's view: High's activity and the occurrence of $\tau$ events. We are in effect asserting that for a non-interfering system all of the non-determinism that Low sees is attributable to the internal $\tau$ activity. Furthermore we see that a corollary of the loose-bisimulation property is that High is noninterfering with the $\tau$ 's, i.e. High cannot influence the occurrence of $\tau$ events. We will see the significance of this observation when we discuss the interaction of flavours of non-determinism in section 3.5.

To return to our simple example, we see that the powerbisimulation relation:

$\{\{P\} \approx\{Q\}$, $\left\{P_{1}^{\prime}, P_{2}^{\prime}\right\} \approx\left\{Q^{\prime}, Q_{1}^{\prime}, Q_{2}^{\prime}\right\}$, $\left.\left\{P_{1}^{\prime \prime}, P_{2}^{\prime \prime}\right\} \approx\left\{Q_{1}^{\prime \prime}, Q_{2}^{\prime \prime}\right\}\right\}$

establishes the power-bisimilarity of the $P$ and $Q$ above.

[3] shows how to construct a power bi-simulation that is is equivalent to failures equivalence. It now suffices to show that the power-bisimulation that we have defined above corresponds to that of the Gardiner approach. Proof of this is beyond the scope of this paper but is presented in a technical report in preparation. The completeness of the unwinding rules, i.e. that they are necessary as well as sufficient thus follows immediately.

The relevance of the notions of bisimulation to defining confidentiality was proposed by Foccardi and Gorrieri in, for example, [2], apparently without reference to analogies with unwinding. The use of the notion of powerbisimulation in security is, to our knowledge, new.

\subsection{Composability}

[14] contains proofs of the composability of this formulation of non-interference with respect to various CSP operators. In fact we see that such composability follows trivially from the composability of failures equivalence (or alternatively from the composability of power bi-simulation) with respect to the CSP operators.

Note however that composability depends on the property and the operators in question. The property of determinacy, for example, defined by Milner in [9] turns out not to be composable with respect to all the CCS operators, which prompts him to introduce the closely associated concept of confluence, which is. Again there appear to be analogies here with some of the quests for composability in the security community and indeed confluence appears to bear some resemblance to the notion of forward correctability. The details of the correspondence are complex however and will not be addressed here.

\subsection{Non-deducibility}

Sutherland's theory of non-deducibility, [18] characterises the lack of information flow in terms of an inability to deduce anything about high level behaviour from a low level view. A system $M$ is said to exhibit this property if every low level view $t r$ of an system execution is compatible with every high level view of inputs $t r^{\prime}$ (in the sense that there is some execution $t r^{\prime \prime}$ which presents both views). This captures the idea that no high-level behaviour can be ruled out by any low-level observation. It is described in process- algebraic terms as follows:

$$
\begin{aligned}
& \forall t r \in \operatorname{traces}\left(M\lceil L), t r^{\prime} \in \operatorname{traces}(M\lceil H I) .\right. \\
& \exists t r^{\prime \prime} \in \operatorname{traces}(M) . \\
& \left(t r^{\prime \prime}\left|L=\operatorname{tr} \wedge t r^{\prime \prime}\right| H I=t r^{\prime}\right)
\end{aligned}
$$

This may also be characterised in a testing framework. A low level user may be considered as testing the system, attempting to elicit information concerning the high level inputs. Thus for non-deducibility to be present, such a low 
level user should not be able to rule out any possibility for $\operatorname{tr}\lceil\mathrm{HI}$-the low level behaviour should be compatible with any high level user inputs.

Suppose that the test $T$ has an alphabet $L$, and some distinguished SUCCESS states. A process may pass a test if there is some execution of the process together with the test which may reach a success state. A process $P$ with its own success states may pass a test $T$ through a system $M$ if there is some execution of $P\|M\| T$ in which both $P$ and $T$ may reach a success state.

Then a system $M$ exhibits non-deducibility if no test $T$ through $M$ may differentiate any two high level users $U 1$ and $U 2$ (drawn from the space of all possible high level users) which have alphabet $H I$, whose traces are only those of $M$ restricted to $H I$, and which have some success states.

The restriction to alphabet $H I$ corresponds to the requirement that any low level information is required to be compatible only with any sequence of high level inputs. This means essentially that no information at the low level is available about the high level input to the system-it is all masked by the system $S$. In other words, for any test $T$ and any two high level users $U 1$ and $U 2, U 1|[H I]| S$ may pass $T$ if and only if $U 2|[H 1]| S$ may pass $T$.

In the state machine formulations of the original definitions, all processes must always be able to accept any input values and provide outputs. This characterises an important subclass of CSP processes. If the space of high level users is restricted to such processes, then the testing formulation is equivalent to Sutherland's. However, CSP allows the consideration of more general classes of process if appropriate.

For example, the system

$$
S=\text { in }_{h} ? x \rightarrow\left(\text { out }_{l} ! 0 \rightarrow S \sqcap \text { out }_{l} ! 1 \rightarrow S\right)
$$

apparently passes no information from high to low. However, the high level process $S T O P$ can be distinguished from $i n_{h} ! 1 \rightarrow$ STOP because a low level test will receive an output when the second is tested, but not when the first is tested. It depends whether STOP is considered a valid description of a high level user or not as to whether $S$ is considered to permit information flow. The characterisation is thus dependent on the space of processes over which the high level users $U$ can range- the kind of activity which must be indistinguishable.

It is observed in [19] that Sutherland's definition has some unwanted consequences. A system meeting this property still permits a high level user to communicate information to a low level user, essentially because high level outputs (which are ignored in the definition) may have a bearing on later high level inputs. It is instructive to recast their example in CSP terms. The system $M\left(k_{1}, k_{2}\right)$ is parametrised by two one-bit keys $k_{1}$ and $k_{2}$. It takes a high level input $h_{i}$, and offers a high level output $h_{o}$ and a low level output $l$. On a high level input $x$ of 0 or 1 , it out- puts that value xor'ed with the key $k_{1}$. Both keys are then randomly reset. On any other high level input (the original example uses a special value $q$ ) the second key $k_{2}$ is output to the low level and randomly reset, and the first key $k_{1}$ is output to the high level.

$$
\begin{aligned}
M\left(k_{1}, k_{2}\right)= & \\
h_{i} ? x \rightarrow & \text { if } x \in\{0,1\} \\
& \text { then } h_{0} ! 0 \rightarrow l !\left(x \oplus k_{1}\right) \rightarrow \\
& \quad \prod_{k_{1}, k_{2} \in\{0,1\}} M\left(k_{1}, k_{2}\right)
\end{aligned}
$$

It is easily checked that this system allows any high level inputs with any low level view, and hence $M$ exhibits nondeducibility.

However, a high level user can use this system to communicate to the low level, by employing a particular strategy. If the high level wishes to transmit a particular bit $b$, then he first learns the value of $k_{1}$ by inputting $q$, and then inputs $k_{1} \oplus b$, which will result in the transmission of $b$ to the low level. Based on this, a transmitter wishing to communicate a sequence $\langle b\rangle^{\frown} u^{\prime}$ of bits behaves as follows:

$$
\begin{aligned}
& T\left(\langle b\rangle \frown u^{\prime}\right)= \\
& \quad h_{i} ! q \rightarrow h_{o} ? k \rightarrow h_{i} !(k \oplus b) \rightarrow h_{o} ? k^{\prime} \rightarrow T\left(u^{\prime}\right)
\end{aligned}
$$

The CSP formulation in terms of $H I$ draws attention to the weak point of Sutherland's definition. The only high level activity it is concerned with is high level input. However, high level users also interact with the system by receiving output, and in this case the output received can influence later input. Thus the definition does not capture all the ways in which the high level user can interact with the system.

The example of [19] can be replaced with a rather more intuitive and immediate example that we believe shows up the essence of the problem.

Consider a system in which high level data is encrypted and the encrypted form transmitted over a low channel. Thus far we have the classic scenario that arises in many security architectures and yet has proved so difficult to capture in a non-interference style framework. Assuming that the encryption scheme is itself 'secure' (in a cryptanalytic sense) then we would tend to accept that such a system is secure.

Suppose now that the high user can in fact observe or predict the key stream before he submits his plain-text for encryption. We can easily imagine this occurring in a number of ways and in fact Wittbold et al's example achieves essentially this. Then High can modify the plain-text in such a way as to communicate it to Low, in particular he can simply xor the predicted bits into the plain-text before 
submitting it to the encryption resulting in raw plain-text being broadcast over the low channel.

This is not too surprising so far but let us examine it more closely as it is a pointer to the root of the problem. The example involves two kinds of non-determinism: external (under the control of the users or processes, in particular High) and probabilistic, arising, in this case, from the cipher stream. In the above example we have allowed these to interact: we have allowed High to resolve his nondeterminism basing his decisions on observations of how the probabilistic non-determinism is being resolved. If we were to force High to resolve all his non-determinism at the outset, before any of the cipher stream has been generated, or in the absence of any knowledge of this source of nondeterminism, then he could not exploit this channel.

We see that this discussion touches on the points made earlier regarding the loose-bisimulation. The loose- bisimulation property implied that High should not be able to interfere with the $\tau$ events. If we can think of the $\tau$ events as representing the non-determinism associated with the cipher stream then we see that this is asserting that High cannot interfere with the cipher stream. The situation is a little delicate as the bisimulation does not prevent flow from the $\tau$ 's to High which potentially could allow the scenario described above. In fact High xor'ing the cipher stream to the data stream is tantamount to interfering with the stream but it is not immediately clear that this has been formally captured. This will be a topic for further investigation. An obvious solution is to also require that the tau's do not interfere with High but this seems a bit heavy handed.

Constructing semantic models for a process algebra to accurately capture the distinction between the various forms of non-determinism has proved difficult. A number of probabilistic process algebras have been proposed, for example Morgan [7] and Lowe [6]. These assume that nondeterminism is resolved at the outset and so do not allow for the possibility of delaying choices and making decisions on the basis of observations of how non-deterministic choices are resolved 'at run time'. We thus see why it has proved so difficult to model this scenario in a non-interference style.

Currently we do not appear to have theories rich enough to fully capture the distinction between the various flavours of non-determinism. Indeed it is not clear that it would be particularly effective to try to develop and apply such extended theories as the greater complexity would probably render them unusable. For the crypto example at least it would seem more effective to use arguments outside the model to establish that the crypto is wholly independent of the rest of the system. In fact a well designed crypto device appropriately incorporated in a secure architecture will have precisely these characteristics: the cipher stream will be unobservable and unpredictable by High (or indeed any other user or process). High may of course be able to deduce the cipher stream after the event if he can observe the cipher-text stream but, crucially, this is too late to exploit. Furthermore an ideal encryption algorithm will have the property that observing an arbitrary length of the stream should not enable further bits to be predicted, i.e. the stream is effectively random. We of course have to take care that any implementations maintain these assumptions. Conventional refinement techniques will not preserve the non-determinism so from a refinement point of view this non-determinism has to be handled differently.

Such a situation is not entirely satisfactory but appears quite workable, at least for a large class of systems. Of course it might be that we encounter systems for which the flavours of non-determinism cannot be so conveniently isolated. This could probably by regarded as a symptom of a poorly designed system.

\subsection{Non-deducibility on strategies}

To avoid the problem with Non-deducibility outlined in the previous section, the notion of Non-deducibility on strategies was introduced [19]. In this formulation, any low view needs to be compatible with any high transmitter strategy, where a strategy describes what a high level user will input depending on previous inputs and outputs. Any low level trace view should thus be compatible with any high level view that is possible for the system.

Non-deducibility on strategies for a system $S$ requires that $(U \| S) \backslash H$ should give the same set of (low level) traces whatever deterministic high level user $U$ is used. (Note that the example $M\left(k_{1}, k_{2}\right)$ above does not have this property.)

Non-deducibility on strategies can be cast in testing terms very similar to the characterisation for nondeducibility above: a system $S$ exhibits non-deducibility on strategies if no test $T$ through $S$ may distinguish between any two high level users who have alphabet $H$. It is essentially a simple variation on Equation 6 .

$$
\begin{aligned}
& \forall t r \in \operatorname{traces}\left(M\lceil L), t r^{\prime} \in \operatorname{traces}(M\lceil H) .\right. \\
& \exists t r^{\prime \prime} \in \operatorname{traces}(M) . \\
& \quad\left(t r^{\prime \prime}\left\lceil L=t r \wedge t r^{\prime \prime} \mid H=t r^{\prime}\right)\right.
\end{aligned}
$$

This definition incorporates all possible high level interactions between the system and the high level users, because they can interact on the entire high level interface $H$ and not simply on $H I$ as in Equation 6. This means essentially that no information at the low level is available about any high level activity of the system at all. In other words, for any test $T$ and any two high level users $U 1$ and $U 2$ meeting the same restrictions as previously, $U 1|[H]| S$ may pass $T$ if and only if $U 2|[H]| S$ may pass $T$. 
When high level users are restricted to the subclass of non-deterministic state machines, then this definition coincides with non-deducibility of strategies. In that case, the system $S$ of equation 7 meets the definition. However, if $U$ can range over all possible CSP processes, then $S$ does not meet the definition: a low level user could distinguish between $S T O P$ and $i n_{h} ! 0 \rightarrow$ STOP.

It is immediate that in the testing formulation Equation 8 implies Equation 6: if a test distinguishes the inputs of two high level users, then the same test will distinguish their high level activity.

\subsection{A deterministic approach to Non-interference}

An alternative definition of non-interference in the process algebra context was proposed by Roscoe, Woodcock and Wulf [12]. In their approach, a system $S$ does not allow information flow from high to low if the system

$$
\text { Abstract }_{H}\left(\text { CHAOS }_{H} \| S\right)
$$

is deterministic. Abstract $_{H}$ denotes an appropriate abstraction of the $\mathrm{H}$ events to obtain a Low view of the system. Typically it will involve a mix of lazy and eager abstraction depending on whether the high events are considered delayable on not. This system presents events only at the low level, and its determinism means that there is only one possibility for each event at any stage (offer, or refusal), however the non-determinism in the system is resolved. Since any high level user will be a refinement of $C H A O S_{H}$, and since all CSP operators preserve the refinement relation, this means that any particular high level user $U_{H}$ in parallel with the system will have to be a refinement of $C H A O S_{H}$ is parallel with the system. Since the result (when $H$ is hidden) is already deterministic, this means that it cannot be further refined so it must remain unchanged. This is true for any high level user $U_{H}$, so all possible high level users must give the same result at the low level. Hence, no low level test can distinguish between them. It follows that this formulation implies non-deducibility on strategies. In a sense, $\mathrm{CHAOS}_{H}$ embodies all possible strategies, and the requirement that the resulting system is deterministic states that all such strategies must always give the same result.

Advantages of this approach are that it side-steps much of the debate about what is the appropriate notion of process equivalence, virtually all the process algebras agree on what processes are deterministic. It is compositional, it is preserved by conventional refinement and it is automatically checkable (using for example using the built in determinism checking facility of FDR).

It is clear that where is can be applied this is an extremely effective characterisation of absence of information flow. The drawback is that there appears to be a large class of systems for which this property is too strong: for which some degree of low-level non-determinism is unavoidable. Perhaps such systems are best handled by isolating such essential non-determinism and showing that the property is preserved by refinement of the rest of the system.

An example will be instructive:

$$
\begin{aligned}
S= & \text { in }_{h} ? x \rightarrow\left(\left(\text { out }_{l} ! 0 \rightarrow S\right) \sqcap\left(\text { out }_{l} ! 1 \rightarrow S\right)\right) \\
& \sqcap\left(\text { out }_{l} ! 0 \rightarrow S\right) \sqcap\left(\text { out }_{l} ! 1 \rightarrow S\right)
\end{aligned}
$$

As it stands, this system does not allow information to flow from the high to the low level. This will remain the case if the non-determinism in the system is essential, and cannot be removed or subverted in some way, (perhaps by a poor implementation of random choice). However, different runs with the same high level user (say $i n_{h} ! 1 \rightarrow S T O P$ ) can result in a number of different possible low level behaviours. Thus $S$ system does not meet definition 9, since it is nondeterministic at the low level.

However, if the system $S$ can be refined when it is implemented, then it is not clear that the result will retain the nondeducibility on strategies. In particular, $S^{\prime}=i n_{h} ? x \rightarrow$ out $_{l} ! x \rightarrow S^{\prime}$ is a refinement of $S$.

If we are dealing with such a situation, where the nondeterminism in the system is not guaranteed to all be retained, or where there is some doubt over its eventual form when the system is implemented, then a definition of noninterference which allows some measure of refinement will be useful. In this case definition 9 is useful, since it is preserved by refinement: any refinement of $S$ will have to result in a refinement of Abstract $_{H}\left(\mathrm{CHAOS}_{H} \| S\right)$, which must therefore be deterministic.

On the other hand, if the random choices in $S$ can be guaranteed to be retained, we might say that the nondeterminism in the system is essential. In this case refinement of $S$ is not a concern and the CSP formulation of nondeducibility on strategies is sufficient to establish a lack of information flow.

It may be that we can establish a link between the determinism approach and the power-bisimulation approach presented in section 3.3. If we think of all the non-determinism observed by Low as resulting from different $\tau$ activities then we could assert that to be non-interfering an abstraction of the system that obscures the High events but retains the $\tau$ 's should be deterministic. This would have many of the advantages of the deterministic approach whilst allowing some non-determinism to manifest itself in Low's view, allowing for example the encrypted channel examples.

The difficulty is that $\tau$ 's are not really part of the CSP framework and that further non-determinism could arise from ambiguous $\tau$ transitions: two distinct transitions from a given state that are both labelled by a $\tau$. Both of these problems can addressed by not labelling them as $\tau$ events but simply regarding them as another set of events hidden from Low and disjoint from High. 


\section{Generalisations of Non-interference}

\subsection{Motivation}

The idea of non-interference is clearly a central one in information security. It is however often argued that in practice it is too strong, that no real policy ever calls for total absence of information flow over any channel and that in any case it is not achievable. For example even the so-called one-way regulators in fact allow for a very low bandwidth feedback from high to low to regulate the upward flow and prevent buffer overflows etc.

Even for the comparatively straightforward MLS style policies simple non-interference runs into problems, for example the encryption problem discussed earlier and the need to incorporate downgrades.

As information processing systems steadily grow more sophisticated and distributed the old paper world analogies look increasingly dated. The demand for security in the commercial sector as well as trends in the military sector are prompting the need for more flexible policies, with a finer granularity of objects and more subtle controls of flows. New paradigms like object orientation, hypertext, virtual machines, mobile code and agents etc allow a far greater granularity of objects to be considered as the elements of a security policy. More sophisticated styles of policy often call for history or location based access decisions and these again cannot be reduced to predicates on the classification labels of individual events or interfaces.

All of these suggests the need to investigate ways in which we might generalise non-interference to allow for partial, conditional flows etc.

\subsection{Formalisation}

We use as our starting point the formulation given in Section 3. The most liberal generalisation of this appears to be:

$$
\begin{aligned}
& \forall t r, t r^{\prime}: \operatorname{traces}(S) . t r \approx t r^{\prime} \Rightarrow \\
& A b s_{H}((S \| \text { Constrain }) / t r) \\
& \quad \equiv A b s_{H}\left(\left(S \| \text { Constrain } / t r^{\prime}\right)\right.
\end{aligned}
$$

where $A b s_{H}$ denotes an appropriate form of abstraction to model the low-level view. We have a number of abstraction operators available to us, for example the various flavours of lazy and eager hiding. Another abstraction that has been used in formalising notions of anonymity but hitherto not confidentiality is 'projection', renaming a set of events to a single event. We will see later how this operator is useful in addressing the encryption problem.

$\approx$ denotes an equivalence relation between behaviours. Hitherto this has typically been defined in terms of some kind of purge function on traces but there seems to be no reason to restrict ourselves to such equivalences.

$\equiv$ denotes an appropriate process equivalence. Which is appropriate depends on the system and policy in question but failures or testing equivalence would seem most likely. In particular using a testing equivalence framework makes it possible to tailor the class of tests to the system and policy in question.

'Constrain' defines a set of high-level behaviours for which we wish to restrict the flow of information. The influence of behaviours of $\mathrm{S}$ that fall outside this envelope on the low-level will be unconstrained by Equation 10. We are regarding them as innocuous and we do not care how they interfere with Low. Presumably a policy might be formulated as the complement of this: stating what behaviours must not interfere, in which case Constrain might not itself be a process, i.e. might not satisfy the closure axioms for a process. How useful a degree of freedom this represents is unclear without trying it out against a sample of policies but we have included it for completeness.

A policy might be encoded as the conjunction of a set of equations of the form of Equation 10.

We can see how various forms of partial or constrained non- interference could be captured in this way. Such a formulation allows Low to determine High's behaviour up to the $\approx$ equivalence. That is, Low can determine which equivalence class High's behaviour belongs to but not where it lies within that class. We can thus define partial information flows and indeed we can arrange to have "non-transitive partial flows': for which information flows from A to B and from $\mathrm{B}$ to $\mathrm{C}$ yet none flows from $\mathrm{A}$ to $\mathrm{C}$.

Consider a data space that can be covered by the coordinates $(x, y)$. Our $A \rightarrow B$ projection loses the $y$ information so projects to $\left(x, y^{\prime}\right)$, for some arbitrary $y^{\prime}$. The $B \rightarrow C$ projection loses the $x$ information so we get $(x, y) \rightarrow\left(x^{\prime}, y\right)$. Putting these two projections together one after the other then gives a projection $A \rightarrow C:(x, y) \rightarrow\left(x^{\prime}, y^{\prime}\right)$, i.e. loss of all information about the original point in the $A$ space.

This example is a bit simplistic as one could easily capture it in a simple labelling formulation. It does serve to illustrate the idea and one can easily construct something more elaborate. A more realistic instance would be a policy that allows for automatic downgrading of certain statistical information from a data base.

Another example in which a more general notion of equivalence seems appropriate is where we consider a high level process editing a file. In general many edit sequences could result in the same final text (we are assuming here that the editor is such that all the details of changes are forgotten and only the final resulting text retained, i.e. there is no mark-up facility). We thus want to regard all edit sequences resulting in the same final text as equivalent. Here again the idea of confluence crops up: that different sequences of ac- 
tions give rise to equivalent states, im particular that certain pairs of actions may be commutative. Thus we might define an equivalence on traces w.r.t. certain permutation groups on actions.

Turning now to the problem of modelling high-level data being encrypted and send out over a low-level channel. Suppose that we take $\approx$ to be defined by:

$t r \approx t r^{\prime} \Leftrightarrow \#(t r \mid H)=\#\left(t r^{\prime} \mid H\right)$

and for our abstraction on the encrypted channel we rename the 0 's and 1's of the enciphered stream to * say. Then we see that $L$ can figure out the length but not the content of the $H$ string. This would appear to accurately model the encrypted channel scenario. We have of course to carefully justify the projection on the cipher channel, presumably using cryptanalytic arguments.

There remains an issue of how to capture the effect of breaches of the crypto system in such a framework, particularly the retrospective effect (previously concealed information is revealed). This remains an open issue, though it does appear to bear a curious resemblance to the downgrading problem.

Notice also that the notion of confidentiality introduced in this example appears to be analogous to some of the notions of anonymity introduced in, for example, [17]. Here of course we are thinking of anonymity over the message space rather than an agent space.

\subsection{Non-transitive non-interference}

Another class of application that is amenable to this approach is that of so-called non-transitive non- interference and channel control policies addressed by Rushby in [13] . A couple of examples are presented for motivation: downgrading and a crypto device. The essence of the problem appears to be that though we want to allow flow from $\mathrm{H}$ to $\mathrm{L}$ it must be regulated, or at the very least audited, by an intermediary. Thus if a high-level data item is to be downgraded and issued to Low this can only happen if accompanied by appropriate actions by a Downgrader process. Similarly high-level data should only be passed to a low-level channel via the crypto device.

Rushby captures this by introducing a more elaborate purge function that, rather than acting in a purely pointwise fashion on the traces, takes account of the affect of downgrade events on the security labels of high events. Clearly we can capture such a policy in our framework with a suitable choice of equivalence, in particular that induced by Rushby's 'ipurge' will do. However we could envisage capturing different forms of intransitive policies to those considered by Rushby by exploiting the full generality of the $\approx$.
A later paper by Pinsky [10] presents an algorithm to construct, where it exists, a minimal equivalence and associated unwinding rule for a downgrading policy. The details are quite complex but it does appear that the algorithm presented by Pinsky can be thought of as an algorithm to construct the appropriate bisimulation relation. Indeed it seems probable that many of the unwinding results presented in the security literature can be interpreted in this way. A number of algorithms for establishing bisimulation relations between putatively equivalent processes are known in the process algebra community and it is probable that they could be usefully applied in the security context.

Note that for a policy encoded in Equation 10 the equivalence relation used can be used to induce a (power) bisimulation relation so establishing an unwinding result. Similarly the composability of such a property follows directly from the composibility of the process equivalence.

\subsection{Encapsulation}

The problem that Rushby addresses in [13] appears to boil down to one of encapsulation: We need to be sure that flows can only occur over certain known channels and that flows that do occur obey certain constraints.

Considering the first aspect: where the process $P$ has no internal sources of non-determinism we can assert that if it looks deterministic over some interface $I$ then $I$ constitutes the entire interface. Where there are internal sources of non-determinism, a process $Z$ say, the problem is more delicate: we presumably need some way of checking that all non-determinism observed over $I$ can be ascribed to $Z$. Naively one could imagine freezing the non-determinism of $Z$, i.e. replacing it by some deterministic implementation. This is not adequate in general however; if there are other sources of non-determinism apart from $Z$ them a particular freezing of $Z$ might eliminate block them whilst other freezings might enable them. This suggests the need to examine all possible freezings but it is not clear that this would really be practical apart from in certain special cases, particularly if we are thinking of applying model-checking techniques. This is a topic of ongoing research.

The second aspect concerns that control of information flows over the channels that have been identified and this appears best done using the framework of equation 10 .

\section{Conclusions/Discussion}

The central thesis of this paper is that the problem of formalising the notion of confidentiality boils down to that of formalising the equivalence of processes. The latter is a central and difficult question at the heart of computer science to which there is no unique answer. Which notion of 
equivalence is appropriate depends on the context and application. Consequently we should not be surprised that the information security community has failed to come up with a consensus on which constitutes confidentiality. Indeed, in this paper we have shown a close correspondence between various proposals for definitions of confidentiality in the security literature and forms of process equivalence in the process algebra literature. Where the system's security can be characterised as the determinism of the low-level view we are in better shape as the definition of determinism is fairly uncontroversial. It is not currently clear how large a class of real systems can be handled in this way.

Viewing security from a process algebraic framework brings with it a number of ready-made results and insights, particularly regarding composition and unwinding. It also helps to pin-point and isolate the source of many of the problems that have been encountered in the security literature, for example regarding the encryption problem, the lack of compositionality of certain formulations etc.

The usefulness of the concept of testing equivalence has also emerged for this viewpoint. This concept seems to have been curiously neglected in the context of defining noninterference though it has recently been used in the analysis of security protocols, see for example Abadi et al, [1]. The philosophy is strikingly similar: in [1] tests can be understood as encapsulating all possible attacks, and equivalence under testing establishes that no such attack can succeed in distinguishing a real system from an ideal one. In our context, tests might be understood as encapsulating all possible ways in which information may flow from high to low, either as a result of High attempting to communicate information, or from the point of view of Low attempting to elicit it, or indeed involving some collusion. Equivalence under testing establishes that no such way can succeed.

It seems very natural to think of two systems as being equivalent if no test can distinguish them. Of course the problem then becomes one of what class of tests is appropriate. In particular the generalised notion of testing introduced in [16] may prove useful in drawing a distinction between delayable and undelayable events at the low-level. Furthermore we have shown the significance of the notion of power-bisimulation. It establishes a correspondence between the testing, bisimulation and denotational styles of defining process equivalence.

We have proposed a generalised form of noninterference and shown that it can encompass a number of systems and policies of interest: with encrypted channels, partial and conditional flows, downgraders etc.

We have only addressed the so-called 'possibilistic' notions of non-interference, i.e. whether High can influence the possibility of Low performing certain observations. In other words we are abstracting away from issues of probability and time, in particular. Clearly these are important and need to be addressed. However it is also clear that even in the comparatively simple context of possibilisitic models there are many subtleties lurking. We hope that this paper has served to identify and shed some more light on these subtleties.

\subsection{Acknowledgements}

Much of the research reported here was carried out during a collaborative visit at Royal Holloway, and the first author is grateful to the Royal Holloway Department of Computer Science for their hospitality. We would also like to thank Bill Roscoe, Michael Goldsmith, Sylvan Pinsky, Paul Gardiner, Martin Abadi and Nick Moffat as well as the reviewers for useful discussions and comments.

\section{References}

[1] M. Abadi and A. Gordon. A calculus for cryptographic protocols: the spi calculus. Information and Computation, 1999.

[2] R. Focardi, A. Ghelli, and R. Gorrieri. Using noninterference for the analysis of security protocols. In DIMACS workshop on Design and Formal Verification of Security protocols, 1997.

[3] P H B Gardiner. Power simulation and its relation to failures refinement. DERA/FSEL technical report, to appear, 1999.

[4] J. A. Goguen and J. Meseguer. Security policies and security models. In IEEE Symposium on Security and Privacy, 1982.

[5] M. Hennessy. Algebraic Theory of Processes. MIT press, 1988.

[6] G. Lowe. Probabilities and Priorities in Timed CSP. D. Phil thesis, Oxford University, 1993.

[7] A. McIver, C. Morgan, K. Seidel, and J. Sanders. Refinement-oriented probability for CSP. Formal Aspects of Computing 8(9), 1996.

[8] J McLean. A general theory of composition for trace sets closed under selective interleaving functions. In IEEE Symposium on Research in Security and Privacy, 1994.

[9] R. Milner. Communication and Concurrency. Prentice-Hall, 1989.

[10] S. Pinsky. Absorbing covers and intransitive noninterference. In IEEE Symposium on Research in Security and Privacy, 1995. 
[11] A. W. Roscoe. The theory and practice of concurrency. Prentice-Hall, 1997.

[12] A. W. Roscoe, J. Woodcock, and L. Wulf. Noninterference through determinism. In ESORICS, 1994.

[13] John Rushby. Noninterference, transitiivity and channel-control security policies. Technical report, SRI, 1992.

[14] P. Y. A. Ryan. A CSP formulation of non-interference and unwinding. Presented at CSFW'90 and published in Cipher Winter90/91, 1990.

[15] S. A. Schneider. Concurrent and Real time systems: the CSP approach. John Wiley, 1999. (to appear).

[16] S. A. Schneider. Testing and abstraction. Technical Report TR-99-02, Royal Holloway, University of London, 1999.

[17] S. A. Schneider and A. Sidiropoulos. CSP and anonymity. In ESORICS, 1996.

[18] D. Sutherland. A model of information. In 9th National Computer Security Conference, 1986.

[19] J. T. Wittbold and D. M. Johnson. Information flow in nondeterministic systems. Proceedings of the 1990 Symposium on Research on Security and Privacy, 1990. 